\title{
GLOBAL ATTRACTORS AND DETERMINING MODES FOR THE 3D NAVIER-STOKES-VOIGHT EQUATIONS
}

\author{
VARGA K. KALANTAROV AND EDRISS S. TITI
}

\begin{abstract}
We investigate the long-term dynamics of the three-dimensional NavierStokes-Voight model of viscoelastic incompressible fluid. Specifically, we derive upper bounds for the number of determining modes for the 3D Navier-Stokes-Voight equations and for the dimension of a global attractor of a semigroup generated by these equations. Viewed from the numerical analysis point of view we consider the Navier-Stokes-Voight model as a non-viscous (inviscid) regularization of the three-dimensional Navier-Stokes equations. Furthermore, we also show that the weak solutions of the Navier- StokesVoight equations converge, in the appropriate norm, to the weak solutions of the inviscid simplified Bardina model, as the viscosity coefficient $\nu \rightarrow 0$.
\end{abstract}

MSC Classification: 37L30, 35Q35, 35Q30, 35B40

Keywords: Navier-Stokes-Voight equations, global attractor, determining modes, regularization of the Navier-Stokes equations, turbulence models, viscoelastic models.

\section{INTRODUCTION}

We consider the three-dimensional Navier-Stokes-Voight (NSV) system of equations

$$
\begin{gathered}
v_{t}-\nu \Delta v-\alpha^{2} \Delta v_{t}+(v \cdot \nabla) v+\nabla p=f(x), x \in \Omega, t \in \mathbb{R}^{+}, \\
\operatorname{div} v=0, x \in \Omega, t \in \mathbb{R}^{+} ; v(x, t)=0, x \in \partial \Omega, t \in \mathbb{R}^{+}, \\
v(x, 0)=v_{0}(x), x \in \Omega,
\end{gathered}
$$

where $\Omega \subset \mathbb{R}^{3}$ is a bounded domain with sufficiently smooth boundary $\partial \Omega, v=v(x, t)$ is the velocity vector field, $p$ is the pressure, $\nu>0$ is the kinematic viscosity, $\alpha$ is a length scale parameter characterizing the elasticity of the fluid, and $f$ is a given force field.

The system (1.1)-(1.2) models the dynamics of a Kelvin-Voight viscoelastic incompressible fluid and was introduced by A.P. Oskolkov in [38] as a model of motion of linear, viscoelastic fluids.

The viscous simplified Bardina model was introduced and studied in [34] (see also [4]) as a simplified version of the Bardina sub-grid scale model of turbulence [3]. In [5] the viscous and inviscid simplified Bardina model were shown to be globally well-posed. It is interesting to observe that the inviscid simplified Bardina model coincides with the inviscid version of the NSV equations (1.1)-(1.3). Viewed from the numerical analysis point of view the authors of [5] proposed the inviscid simplified Bardina model (or equivalently the inviscid NSV equations) as a non-viscous (inviscid) regularization of the 3D Euler

Date: May 27, 2007. 
equations, subject to periodic boundary conditions. Motivated by this observation the system (1.1)-(1.3) was also proposed in [5] as a regularization, for small values of $\alpha$, of the 3D Navier-Stokes (NS) equations for the purpose of direct numerical simulations for both the periodic and the no-slip Dirichlet boundary conditions.

In [38] it is shown that the initial boundary value problem (1.1)-(1.3) has a unique weak solution. In [25] and [26] it is shown that the semigroup generated by the problem (1.1)-(1.3) has a finite dimensional global attractor.

In this paper we give an estimate of the fractal and Hausdorff dimensions of the global attractor of a dynamical system generated by the problem (1.1)-(1.3), which is an improvement of the estimates done in [26]. Moreover, we derive estimates for the number of asymptotic determining modes of the solutions of the problem (1.1)-(1.3). We also show that there exists a number $m$ such that each trajectory $v(t)$ on the global attractor of the dynamical system generated by this problem is uniquely determined by its projection $P_{m} v(t)$ onto the $\operatorname{span}\left\{w_{1}, \ldots, w_{m}\right\}$ of the first $m$ eigenfunctions of the Stokes operator. This observation is related to the notion of continuous data assimilations as it has been presented in [29, [36] and [37].

It is worth stressing that by adding the regularizing term $\left(-\alpha \Delta v_{t}\right)$ to the NS equations the system (1.1)-(1.3) changes its parabolic character. In particular, the 3D system (1.1)(1.3) is globally well-posed forward and backwards in time. The semigroup generated by the problem (1.1)-(1.3) is only asymptotically compact. In this sense the system is similar to damped hyperbolic systems. We also remark that this type of inviscid regularization has been recently used for the two-dimensional surface quasi-geostropic model [28]. In particular, necessary and sufficient conditions for the formation of singularity were presented in terms of regularizing parameter.

\section{Preliminary}

In this paper we will be using the following standard notations in the mathematical theory of NS equations:

- $L^{p}(\Omega), 1 \leq p \leq \infty$, and $H^{s}(\Omega)$ are the usual Lebesgue and Sobolev spaces, respectively.

- For $v=\left(v_{1}, v_{2}, v_{3}\right)$, and $u=\left(u_{1}, u_{2}, u_{3}\right)$ we denote by

$$
(u, v)=\sum_{j=1}^{3}\left(v_{j}, u_{j}\right)_{L^{2}(\Omega)},\|v\|^{2}=\sum_{j=1}^{3}\left\|v_{i}\right\|_{L^{2}(\Omega)}^{2},\|\nabla v\|^{2}:=\sum_{j, i=1}^{3}\left\|\partial_{i} v_{j}\right\|_{L^{2}(\Omega)}^{2} .
$$

- We set

$$
\mathcal{V}:=\left\{v \in\left(C_{0}^{\infty}(\Omega)\right)^{3}: \nabla \cdot v=0\right\} .
$$

- $H$ is the closure of the set $\mathcal{V}$ in $\left(L_{2}(\Omega)\right)^{3}$ topology.

- $\mathrm{P}$ is the Helmholz-Leray orthogonal projection in $\left(L^{2}(\Omega)\right)^{3}$ onto the space $H$, and $h:=P f$. 
- $A:=-P \Delta$ is the Stokes operator subject to the no-slip homogeneous Dirichlet boundary condition with the domain $\left(H^{2}(\Omega)\right)^{3} \cap V$. The operator $A$ is a selfadjoint positively definite operator in $H$, whose inverse $A^{-1}$ is a compact operator from $H$ into $H$. Thus it has an orthonormal system of eigenfunctions $\left\{w_{j}\right\}_{j=1}^{\infty}$ of $A$.

- We denote by $\left\{\lambda_{j}\right\}_{j=1}^{\infty}, 0<\lambda_{1} \leq \lambda_{2} \leq \cdots$, the eigenvalues of the Stokes operator $A$ corresponding to eigenfunctions $\left\{w_{j}\right\}_{j=1}^{\infty}$, repeated according to their multiplicities.

- $V_{s}:=D\left(A^{s / 2}\right),\|v\|_{s}:=\left\|A^{s / 2} v\right\|, s \in \mathbb{R} . V:=V_{1}=\left(H_{0}^{1}(\Omega)\right)^{3} \cap H$ is the Hilbert space with the norm $\|v\|_{1}=\|u\|_{V}=\|\nabla u\|$, thanks to the Poincaré inequality (2.3). Clearly $V_{0}=H$.

- For $u, v, w \in \mathcal{V}$ we define the following bilinear form

$$
B(u, v):=P((u \cdot \nabla) v) \text { and the trilinear form } b(u, v, w)=(B(u, v), w) .
$$

The bilinear form $B(\cdot, \cdot)$ can be extended as a continuous operator $B: V \times V \rightarrow V^{\prime}$, where $V^{\prime}$ is the dual of $V$ (see, e.g., [11]).

- For each $u, v, w \in V$

$$
b(u, v, v)=0 \text {, and } b(u, v, w)=-b(u, w, v) .
$$

Next we formulate some well known inequalities and a Gronwall type lemma that we will be using in what follows.

\section{Young's inequality}

$$
a b \leq \frac{\varepsilon}{p} a^{p}+\frac{1}{q \varepsilon^{1 /(p-1)}} b^{q}, \text { for all } a, b, \varepsilon>0, \text { with } q=p /(p-1), 1<p<\infty .
$$

Poincaré inequality

$$
\|u\| \leq \lambda_{1}^{-1 / 2}\|u\|_{1}, \quad \forall u \in V
$$

where $\lambda_{1}$ is the first eigenvalue of the Stokes operator under the homogeneous Dirichlet boundary condition.

Hereafter, $C$ will denote a dimensionless scale invariant constant which might depend on the shape of the domain $\Omega$.

Ladyzhenskaya inequalities ([11], [31], [33])

$$
\begin{gathered}
\|u\|_{L^{3}} \leq C\|u\|^{1 / 2}\|\nabla u\|^{1 / 2}, \forall u \in V, \\
\|u\|_{L^{4}} \leq C\|u\|^{1 / 4}\|u\|_{1}^{3 / 4}, \quad \forall u \in V .
\end{gathered}
$$

Sobolev inequality (see, e.g., [1])

$$
\|u\|_{L^{6}} \leq C\|u\|_{1}, \forall u \in V .
$$

Gagliardo - Nirenberg inequalities (see, e.g., [2, [11],[33])

$$
\|u\|_{L^{6 /(3-2 \varepsilon)}} \leq C\|u\|^{1-\varepsilon}\|u\|_{1}^{\varepsilon}, \quad 0 \leq \varepsilon \leq 1, \quad \forall u \in V .
$$




$$
\|u\|_{L^{p}} \leq C\|u\|^{2 / p}\|u\|_{3 / 2}^{1-2 / p}, \quad p \in[2, \infty), \quad \forall u \in V_{3 / 2} .
$$

Agmon inequality (see, e.g., [11])

$$
\|u\|_{L^{\infty}(\Omega)} \leq C\|u\|_{1}^{1 / 2}\|A u\|^{1 / 2}, \forall u \in V_{2}
$$

We will use also the following estimates of the trilinear form $b(u, v, w)$ which follow from (2.4) - (2.9) (see, e.g., [11]).

$$
\begin{gathered}
|b(u, v, w)| \leq C\|u\|^{1 / 2}\|u\|_{1}^{1 / 2}\|v\|_{1}\|w\|_{1}, \quad \forall u, v, w \in V, \\
|b(u, v, u)| \leq C\|u\|^{1 / 2}\|u\|_{1}^{3 / 2}\|v\|_{1}, \quad \forall u, v \in V, \\
|b(u, v, w)| \leq C\|u\|_{1}\|v\|_{1}\|w\|^{1 / 2}\|w\|_{1}^{1 / 2}, \quad \forall u, v, w \in V, \\
|b(u, v, w)| \leq C \lambda_{1}^{1 / 4}\|u\|_{1}\|v\|_{1}\|w\|_{1}, \quad \forall u, v, w \in V .
\end{gathered}
$$

Lemma 2.1. (23], see also [15]) Let $a(t)$ and $b(t)$ be locally integrable functions on $(0, \infty)$ which satisfy for some $T>0$ the conditions

$$
\liminf _{t \rightarrow \infty} \frac{1}{T} \int_{t}^{t+T} a(\tau) d \tau=\gamma, \limsup _{t \rightarrow \infty} \frac{1}{T} \int_{t}^{t+T} a^{-}(\tau) d \tau=\Gamma, \liminf _{t \rightarrow \infty} \frac{1}{T} \int_{t}^{t+T} b^{+}(\tau) d \tau=0,
$$

where $\gamma>0, \Gamma<\infty, a^{-}=\max \{-a, 0\}$ and $b^{+}=\max \{b, 0\}$. If a non-negative, absolutely continuous function $\phi(t)$, satisfies

$$
\phi^{\prime}(t)+a(t) \phi(t) \leq b(t), t \in(0, \infty)
$$

then $\phi(t) \rightarrow 0$ as $t \rightarrow \infty$.

Definition 2.2. (see, e.g., [15], [19], [32]) A semigroup $S(t): V \rightarrow V, t \geq 0$ is called asymptotically compact, if for any sequence of positive numbers $t_{n} \rightarrow \infty$ and any bounded sequence $\left\{v_{n}\right\} \subset V$ the sequence $\left\{S\left(t_{n}\right) v_{n}\right\}$ is precompact in $V$.

Theorem 2.3. (see, e.g., [19], [32, [43]) Assume that a semigroup $S(t): V \rightarrow V$, for $t \geq t_{0}>0$ can be decomposed into the form

$$
S(t)=Y(t)+Z(t),
$$

where $Z(t)$ is a compact operator in $V$ for each $t \geq t_{0}>0$. Assume also that there is a continuous function $k:\left[t_{0}, \infty\right) \times \mathbb{R}^{+} \rightarrow \mathbb{R}^{+}$such that for every $R>0 k(t, R) \rightarrow 0$ as $t \rightarrow \infty$ and

$$
\|Y(t) v\|_{V} \leq k(t, R), \quad \text { for all } t \geq t_{0}>0, \quad \text { and all }\|v\|_{V} \leq R .
$$

Then $S(t): V \rightarrow V, t \geq 0$ is asymptotically compact.

Next we state a result from [32] which will enable us to estimate the dimension of the global attractor for the system (1.1)-(1.3). This result is typically useful in the context of nonlinear damped hyperbolic systems, when the damping term is not strong enough to control the instabilities rising from the perturbed nonlinearity. 
Theorem 2.4. (see [13], [32]) Let $S(t), t \in \mathbb{R}^{+}$, be a semigroup generated by the problem

$$
v_{t}(t)=\Phi(v(t)),\left.\quad v\right|_{t=0}=v_{0}
$$

in the phase space $H$ and let $\mathcal{M} \subset H$ is a compact invariant subset with respect to $S(t)$. Let $S(t)$ and $\Phi(\cdot)$ be uniformly differentiable on $\mathcal{M}$ and let $L\left(t, v_{0}\right)$ be a differential of $\Phi$ at the point $S(t) v_{0}, v_{0} \in \mathcal{M}$. Suppose that $L^{c}\left(t, v_{0}\right):=L\left(t, v_{0}\right)+L^{*}\left(t, v_{0}\right), v_{0} \in \mathcal{M}$ satisfies the inequality

$$
\left(L^{c}(t) u, u\right) \leq-h_{0}(t)\|u\|^{2}+\sum_{k=1}^{m} h_{s_{k}}(t)\|u\|_{s_{k}}^{2},
$$

for some numbers $s_{k}<0,(k=1, \ldots, m)$ and some functions $h_{0}, h_{s_{k}} \in L_{1, l o c}(\mathbb{R}), h_{s_{k}}(t) \geq$ $0, h_{0}(t) \geq 0$ for all $t \in \mathbb{R}^{+}$.

Then

$$
\operatorname{dim}_{\mathcal{H}}(\mathcal{M}) \leq \operatorname{dim}_{f}(\mathcal{M}) \leq N
$$

where $N$ is such that

$$
-\bar{h}_{0}(T)+\sum_{k=0}^{m} \bar{h}_{s_{k}}(T) N^{s_{k}}<0,
$$

for some $T>0$. Here $\bar{h}_{i}(T):=\frac{1}{T} \int_{0}^{T} h_{i}(\tau) d \tau$.

\section{Existence of Global Attractors}

Applying the Helmholtz - Leray projector $P$ to the system (1.1)-(1.2), we obtain the following equivalent functional differential equation

$$
\begin{gathered}
v_{t}+\nu A v+\alpha^{2} A v_{t}+B(v, v)=h, \quad h=P f, \\
v(0)=v_{0} .
\end{gathered}
$$

The question of global existence and uniqueness of (3.1)-(3.2) first was studied in [38], where actually it was established that the problem (1.1)-(1.3) generates a continuous semigroup $S(t): V \rightarrow V, t \in \mathbb{R}^{+}$. In [5] the authors proved also the global regularity for inviscid model of (3.1), i.e. when $\nu=0$.

In this section we show that the semigroup $S(t)$ generated by the problem (1.1)-(1.3) has an absorbing ball in $V$ and an absorbing ball in $V_{2}$. Then we show that $S(t): V \rightarrow V$, for $t \in \mathbb{R}^{+}$is an asymptotically compact semigroup, and deduce the existence of a global attractor in $V$.

Let us note that the formal estimates we provide below can be justified rigorously by using a Galerkin approximation procedure and passing to the limit, by using the relevant Aubin's compactness theorem as for the NS equations ( see, for example, [11, [15], [41] or [43]).

Absorbing ball in $V$. Taking the inner product of (3.1) with $v$, and noting that due to (2.1) $(B(v, v), v)=0$, we get

$$
\frac{d}{d t}\left[\|v(t)\|^{2}+\alpha^{2}\|v(t)\|_{1}^{2}\right]+2 \nu\|v(t)\|_{1}^{2} \leq 2\|h\|_{-1}\|v(t)\|_{1} .
$$


It is easy to see by Poincaré inequality (2.3) that

$$
\nu\|v(t)\|_{1}^{2} \geq \frac{\nu}{2}\left[\lambda_{1}\|v\|^{2}+\|v(t)\|_{1}^{2}\right] \geq d_{0}\left[\|v(t)\|^{2}+\alpha^{2}\|v(t)\|_{1}^{2}\right],
$$

where $d_{0}:=\frac{\nu}{2} \min \left\{\frac{1}{\alpha^{2}}, \lambda_{1}\right\}=\nu d_{1}$. Hence (3.3) implies

$$
\frac{d}{d t}\left[\|v(t)\|^{2}+\alpha^{2}\|v(t)\|_{1}^{2}\right]+d_{0}\left[\|v(t)\|^{2}+\alpha^{2}\|v(t)\|_{1}^{2}\right] \leq \frac{1}{\nu}\|h\|_{-1}^{2}
$$

By Gronwall's inequality we have

$$
\begin{gathered}
\|v(t)\|^{2}+\alpha^{2}\|v(t)\|_{1}^{2} \leq \\
e^{-d_{0}(t-s)}\left[\|v(s)\|^{2}+\alpha^{2}\|v(s)\|_{1}^{2}-\frac{\|h\|_{-1}^{2}}{\nu d_{0}}\right]+\frac{1}{\nu d_{0}}\|h\|_{-1}^{2} .
\end{gathered}
$$

Therefore,

$$
\limsup _{t \rightarrow \infty}\left[\|v(t)\|^{2}+\alpha^{2}\|v(t)\|_{1}^{2}\right] \leq \frac{\|h\|_{-1}^{2}}{\nu d_{0}} .
$$

The last inequality implies that the semigroup $S(t): V \rightarrow V, t \in \mathbb{R}^{+}$generated by the problem (1.1)-(1.3) (or equivalently (3.1) $-(\underline{3.2})$ ) has an absorbing ball

$$
\mathcal{B}_{1}:=\left\{v \in V:\|v\|_{1} \leq \frac{2}{\sqrt{\nu \alpha^{2} d_{0}}}\|h\|_{-1}\right\} .
$$

Hence, the following uniform estimate is valid

$$
\|v(t)\|_{1} \leq M_{1}
$$

where $M_{1}=\frac{2}{\nu \alpha \sqrt{d_{1}}}\|h\|_{-1}$, for $t$ large enough $(t \gg 1)$ depending on the initial data.

Asymptotic compactness. By using the Galerkin procedure it is not difficult to prove the following

Proposition 3.1. Let $s \in \mathbb{R}$. If $w_{0} \in V_{s}, g \in L^{2}\left([0, T) ; V_{s-2}\right)$ then the linear problem

$$
z_{t}+\alpha^{2} A z_{t}+\nu A z=g(t), z(0)=0
$$

has a unique weak solution which belongs to $C\left([0, T) ; V_{s}\right)$ and the following inequality holds

$$
\sup _{t \in[0, T)}\|z(t)\|_{s} \leq C\|g\|_{L^{2}\left(0, T ; V_{s-2}\right)}, s \in \mathbb{R} .
$$

Proposition 3.2. Let $h \in H$, be time independent, then the semigroup $S(t), t \geq 0$ is asymptotically compact semigroup in $V$.

Proof. Let $v_{0} \in V$. First we observe that $S(t)$ has the representation

$$
S(t) v_{0}=Y(t) v_{0}+Z(t) v_{0}
$$

where $Y(t)$ is the semigroup, generated by the linear problem

$$
y_{t}+\nu A y+\alpha^{2} A y_{t}=0, y(0)=v_{0}
$$

and $z(t)=Z(t)\left(v_{0}\right)$ is the solution of the problem

$$
z_{t}+\nu A z+\alpha^{2} A z_{t}=h-B(v(t), v(t)), z(0)=0,
$$


where $v$ is the solution of (1.1)-(1.3) (or equivalently (3.1)-(3.2)) with the initial data $v_{0}$. Taking the $H$ inner product of (3.8) with $y$ we obtain

$$
\frac{d}{d t}\left[\|y(t)\|^{2}+\alpha^{2}\|y(t)\|_{1}^{2}\right]+d_{0}\left[\|y(t)\|^{2}+\alpha^{2}\|y(t)\|_{1}^{2}\right] \leq 0,
$$

where we recall that $d_{0}=\nu d_{1}=\nu \frac{1}{2} \min \left\{\frac{1}{\alpha^{2}}, \lambda_{1}\right\}$.

This inequality implies that

$$
\|y(t)\|^{2}+\alpha^{2}\|y(t)\|_{1}^{2} \leq e^{-d_{0} t}\left[\left\|v_{0}\right\|^{2}+\alpha^{2}\left\|v_{0}\right\|_{1}^{2}\right] \text {, for all } t>0 .
$$

So the semigroup $Y(t): V \rightarrow V$ is exponentially contractive.

Due to Hölder's inequality and the Sobolev inequality (2.6) we have

$$
\begin{aligned}
& \|B(v, v)\|_{-1 / 2}=\sup _{\phi \in V,\left\|A^{1 / 4} \phi\right\|=1} b(v, v, \phi)= \\
& \sup _{\phi \in V,\left\|A^{1 / 4} \phi\right\|=1} \int_{\Omega} P((v \cdot \nabla) v) \cdot \phi d x=\sup _{\phi \in V,\left\|A^{1 / 4} \phi\right\|=1} \int_{\Omega}(v \cdot \nabla) v \cdot P \phi d x= \\
& \sup _{\phi \in V,\left\|A^{1 / 4} \phi\right\|=1} \int_{\Omega}(v \cdot \nabla) v \cdot \phi d x \leq C \sup _{\phi \in V,\left\|A^{1 / 4} \phi\right\|=1}\|v\|_{L^{6}}\|v\|_{1}\|\phi\|_{L^{3}} .
\end{aligned}
$$

Hence due to the Sobolev inequality $\|\phi\|_{L^{3}} \leq C\left\|A^{1 / 4} \phi\right\|$ and (2.6) we have

$$
\|B(v, v)\|_{-1 / 2} \leq C \sup _{\phi \in V,\left\|A^{1 / 4} \phi\right\|=1}\|v\|_{1}^{2}\left\|A^{1 / 4} \phi\right\| \leq C\|v\|_{1}^{2},
$$

and

$$
B(v, v) \in L^{\infty}\left(\mathbb{R}^{+} ; V_{-1 / 2}\right) .
$$

The function $v(t)$ as a solution of the problem (3.1)-(3.2) with $v_{0} \in V$ belongs to $L^{\infty}\left(\mathbb{R}^{+} ; V\right)$. Thus due to the inequality (3.11) and the Proposition [3.1, the solution of the problem (3.9) belongs to $C\left(\mathbb{R}^{+} ; V_{3 / 2}\right)$, that is the operator $Z(t)$ maps $V$ into $V_{3 / 2}$. Since the embedding $V_{3 / 2} \subset V$ is a compact embedding, the operator $Z(t)$ is a compact operator for each $t>0$. Hence, the semigroup $S(t)$ satisfies the conditions of the Theorem 2.3, and is an asymptotically compact semigroup.

Since each bounded dissipative and asymptotically compact semigroup possesses a compact global attractor (see, e.g., [2], [19], [31], [43]) we have:

Theorem 3.3. If $h \in H$ then the semigroup $S(t): V \rightarrow V$ has an absorbing ball $\mathcal{B}_{1}=$ $\left\{v \in V:\|v\|_{1} \leq M_{1}\right\}$ and a global attractor $\mathcal{A}_{1} \subset V$. The attractor $\mathcal{A}_{1}$ is compact, connected and invariant.

Next we show that the global attractor $\mathcal{A}_{1}$ is a bounded subset of $V_{2}$.

Taking the inner product in $V_{1 / 2}$ of the equation (3.9) with $z$, and remembering that $v(t)=y(t)+z(t) \in \mathcal{A}_{1}$, we get

$$
\begin{aligned}
\frac{d}{d t}\left[\|z(t)\|_{1 / 2}^{2}+\alpha^{2}\|z(t)\|_{3 / 2}^{2}\right]+2 \nu\|z(t)\|_{3 / 2}^{2} & = \\
& 2(h, z(t))_{1 / 2}-2(B(v(t), v(t)), z(t))_{1 / 2} .
\end{aligned}
$$


The first term on the right-hand side has the estimate

$$
\left|2(h, z(t))_{1 / 2}\right| \leq 2\|h\|_{-1 / 2}\|z(t)\|_{3 / 2} \leq \frac{\nu}{2}\|z(t)\|_{3 / 2}^{2}+\frac{2}{\nu}\|h\|_{-1 / 2}^{2} .
$$

The second term, due to (3.11), has the following estimate

$$
\begin{aligned}
\left|2(B(v(t), v(t)), z(t))_{1 / 2}\right| \leq & C \|\left(B(v(t), v(t))\left\|_{-1 / 2}\right\| z(t) \|_{3 / 2} \leq\right. \\
& \frac{\nu}{2}\|z(t)\|_{3 / 2}^{2}+\frac{C}{\nu}\|B(v(t), v(t))\|_{-1 / 2}^{2} \leq \frac{\nu}{2}\|z(t)\|_{3 / 2}^{2}+\frac{C}{\nu}\|v\|_{1}^{4} .
\end{aligned}
$$

Taking into account the last two inequalities in (3.12) we obtain

$$
\begin{aligned}
\frac{d}{d t}\left[\|z(t)\|_{1 / 2}^{2}+\alpha^{2}\|z(t)\|_{3 / 2}^{2}\right]+2 d_{0}\left[\|z(t)\|_{1 / 2}^{2}+\alpha^{2}\|z(t)\|_{3 / 2}^{2}\right] \leq & \\
& \frac{C}{\nu}\left(\|v(t)\|_{1}^{4}+\|h\|_{-1 / 2}^{2}\right) .
\end{aligned}
$$

Integrating the last inequality we obtain the estimate

$$
\|z(t)\|_{3 / 2}^{2} \leq \frac{C}{d_{0} \alpha^{2} \nu}\left(M_{1}^{4}+\|h\|_{-1 / 2}^{2}\right)=L_{0}
$$

Since the attractor $\mathcal{A}_{1}$ is invariant, $S(t) \mathcal{A}_{1}=\mathcal{A}_{1}$, and due to (3.10) the inequality

$$
\|v(t)-z(t)\|_{1}=\|y(t)\|_{1} \leq C\left(\|y(0)\|_{1}\right) e^{-d_{0} t}
$$

holds, we deduce that for each $u \in \mathcal{A}_{1}$ there exists a sequence $\left\{z\left(t_{k}\right)\right\}, t_{k} \rightarrow \infty$, corresponding to $v_{k}(0) \in \mathcal{A}_{1}$, such that

$$
u=\lim _{k \rightarrow \infty} z\left(t_{k}\right), v_{k}(0) \in \mathcal{A}_{1} .
$$

Thanks to (3.13) the sequence $\left\{z\left(t_{k}\right)\right\}$ is belonging to a ball in $V_{3 / 2}$, whose radius $L_{0}$ depends only on $M_{1}$ and $\|h\|$. Hence, the sequence $\left\{z\left(t_{k}\right)\right\}$ is weakly compact in $V_{3 / 2}$. Thus, by using (3.14) and the inequality $\|u\|_{3 / 2} \leq \liminf _{t_{k} \rightarrow \infty}\left\|z\left(t_{k}\right)\right\|_{3 / 2}$, we see that $\mathcal{A}_{1}$ is bounded in $V_{3 / 2}$.

Knowing that $\mathcal{A}_{1}$ is bounded in $V_{3 / 2}$ we can use similar arguments to show that $\mathcal{A}_{1}$ is also bounded in $V_{5 / 3}$ and in $V_{2}$.

$V_{2}$ absorbing ball. To show that the semigroup $S(t): V_{2} \rightarrow V_{2}$ has an absorbing ball in the phase space $V_{2}=D(A)$ we take $H$ inner product of (3.1) with $A v(t)$ :

$$
\frac{d}{d t}\left[\|v(t)\|_{1}^{2}+\alpha^{2}\|A v(t)\|^{2}\right]+2 \nu\|A v(t)\|^{2}+2(B(v(t), v(t)), A v(t))=2(h, A v(t)) .
$$

For the first term in the right hand side of (3.15) we have

$$
|2(h, A v(t))| \leq \frac{1}{\nu}\|h\|^{2}+\nu\|A v(t)\|^{2} .
$$


By using the Agmon's inequality (2.9) and Young's inequality (2.2) with $p=4 / 3$ we can estimate the last term in the left-hand side of (3.15) as follows

$$
\begin{aligned}
2|(B(v, v), A v)| \leq C\|v\|_{L^{\infty}(\Omega)}\|\| v\left\|_{1}\right\|\|A v\| \leq C\|v\|_{1}^{3 / 2}\|\| A v \|^{3 / 2} \leq & \\
& \frac{3}{4} \epsilon\|A v\|^{2}+\frac{C}{\epsilon^{3}}\|v\|_{1}^{6} .
\end{aligned}
$$

Employing (3.16) and the last inequality, with $\epsilon=2 \nu / 3$, we obtain from (3.15)

$$
\frac{d}{d t}\left[\|v(t)\|_{1}^{2}+\alpha^{2}\|A v(t)\|^{2}\right]+\nu\|A v(t)\|^{2} \leq \frac{1}{\nu}\|h\|^{2}+\frac{C}{\nu^{3}}\|v(t)\|_{1}^{6} .
$$

It follows from (3.17) that

$$
\frac{d}{d t}\left[\|v(t)\|_{1}^{2}+\alpha^{2}\|A v(t)\|^{2}\right]+d_{0}\left[\|v(t)\|_{1}^{2}+\alpha^{2}\|A v(t)\|^{2}\right] \leq \frac{1}{\nu}\|h\|^{2}+\frac{C}{\nu^{3}}\|v(t)\|_{1}^{6} .
$$

Let $t_{0}$ be so that (3.5) holds for all $t \geq t_{0}$. Then integrating the last inequality over the interval $\left(t_{0}, t\right)$ we get

$$
\begin{aligned}
\|v(t)\|_{1}^{2}+\alpha^{2}\|A v(t)\|^{2} & \leq \\
& \quad\left[\left\|v\left(t_{0}\right)\right\|_{1}^{2}+\alpha^{2}\left\|A v\left(t_{0}\right)\right\|^{2}\right] e^{-d_{0}\left(t-t_{0}\right)}+\frac{R_{2}}{d_{0}}\left(1-e^{-d_{0}\left(t-t_{0}\right)}\right),
\end{aligned}
$$

where $R_{2}:=\frac{1}{\nu}\|h\|^{2}+\frac{C}{\nu^{3}} M_{1}^{6}$.

The last inequality implies existence of an absorbing ball

$$
\mathcal{B}_{2}:=\left\{v \in V_{2}:\|A v\| \leq M_{2}\right\}
$$

where $M_{2}^{2}=\frac{2 R_{2}}{\left(\alpha^{2}+\lambda_{1}^{-1}\right) d_{0}}$. That is, for all $t>>1$, we have $\|A v(t)\| \leq M_{2}$.

Similarly, we can prove the following theorem

Theorem 3.4. If $h \in V_{1}$, then the semigroup $S(t): V_{2} \rightarrow V_{2}$ has a global attractor $\mathcal{A}_{2} \subset V_{2}$. The attractor $\mathcal{A}_{2}$ is compact, connected and invariant. Moreover, $\mathcal{A}_{2}$ is a bounded set in $V_{3}$.

Remark 3.5. Let us note that in case we assume in Theorem 3.3 that $h \in V_{1}$, instead of $h \in H$, then the attractors $\mathcal{A}_{1}$ and $\mathcal{A}_{2}$ coincide.

\section{Estimates for the Number of Determining Modes}

It is asserted, based on physical heuristic arguments, that the long-time behavior of turbulent flows is determined by a finite number degrees of freedom. This concept was formulated more rigorously for 2D NS equations by introducing the notion of determining modes in [17]. In [17] it was shown that there exists a number $m$ such that if the first $m$ Fourier modes of two different solutions of the NS equations have the same asymptotic behavior, as $t \rightarrow \infty$, then the remaining infinitely many number of modes have the same asymptotic behavior. 
In [31] it was shown that the semigroup generated by the initial boundary value problem for the 2D NS equations with Dirichlet boundary condition has a global attractor which is compact, invariant and connected. It was also established in [31] that there exists a number $m$ such that if projections of two different trajectories on the attractor on the $m$ dimensional subspace of $H$, spanned on the first $m$ eigenfunctions of the Stokes operator, coincide for each $t \in \mathbb{R}$, then these trajectories completely coincide for each $t \in \mathbb{R}$.

The results obtained in [17] and [31] were developed, generalized, and applied to various infinite dimensional dissipative problems (see, e.g., [7], [8], [9], [15, [16], [18], [20], [22], [23], 24], [32], 36], 37] and references therein).

In this section we are going to give estimates for the number of determining modes (both asymptotic and for trajectories on the attractor) for 3D NSV equations.

Asymptotic determining modes. Let us denote by $P_{m}$ the $L^{2}$-orthogonal projection from $H$ onto the $m$ - dimensional subspace $H_{m}=$ span $\left\{w_{1}, w_{2}, \ldots, w_{m}\right\}$. We set $Q_{m}=$ $I-P_{m}$.

Let $v$ and $u$ be two solutions of NSV equations

$$
\begin{aligned}
& v_{t}+\nu A v+\alpha^{2} A v_{t}+B(v, v)=h(t), v(0)=v_{0}, \\
& u_{t}+\nu A v+\alpha^{2} A u_{t}+B(u, u)=g(t), v(0)=v_{0} .
\end{aligned}
$$

Definition 4.1. A set of modes $\left\{w_{1}, \cdots, w_{m}\right\}$ is called asymptotically determining (see [15],[17]) if

$$
\lim _{t \rightarrow \infty}\|v(t)-u(t)\|_{1}=0
$$

whenever

$$
\lim _{t \rightarrow \infty}\|h(t)-g(t)\|_{-1}=0 \text { and } \lim _{t \rightarrow \infty}\left\|P_{m}(v(t)-u(t))\right\|_{1}=0 .
$$

Theorem 4.2. Assume that the following conditions are satisfied

$$
\begin{gathered}
\|h(t)\|_{-1} \leq \mathbf{h}<\infty, \forall t \in \mathbb{R} . \\
\lim _{t \rightarrow \infty}\|h(t)-g(t)\|_{-1}=0 \text { and } \lim _{t \rightarrow \infty}\left\|P_{m}(v(t)-u(t))\right\|=0 .
\end{gathered}
$$

Then the first $m$ eigenfunctions of the Stokes operator are asymptotically determining for the NSV equations with homogeneous Dirichlet boundary conditions, provided $m$ is large enough such that

$$
\lambda_{m+1}>C \frac{\mathbf{h}^{4}}{\alpha^{4} \nu^{8} d_{1}^{2}} .
$$

Proof. It is clear that the function $w=v-u$ satisfies

$$
w_{t}+\nu A w+\alpha^{2} A w_{t}+B(v, w)+B(w, v)-B(w, w)=\theta(t), v(0)=v_{0},
$$

where $\theta(t)=h(t)-g(t)$. 
It is clear from the proof of $\left(E_{1}\right)$ that

$$
\limsup _{t \rightarrow \infty}\|v(t)\|_{1} \leq \frac{\mathbf{h}}{\alpha \nu \sqrt{d_{1}}} .
$$

Multiplying (4.6) by $q(t)=Q_{m} w(t)$ in $H$ we obtain

$$
\begin{aligned}
& \frac{d}{d t}\left[\|q\|^{2}+\alpha^{2}\|q\|_{1}^{2}\right]+2 \nu\|q\|_{1}^{2}+2 b(q, v, q)= \\
& 2(\theta, q)-2 b(v, p, q)-2 b(p, p, q)+2 b(q, p, q),
\end{aligned}
$$

where $p=P_{m} w$.

Before estimating the terms of (4.8) we observe that for each $\phi \in V$ we have

$$
\left\|Q_{m} \phi\right\|_{1} \geq \lambda_{m+1}\left\|Q_{m} \phi\right\| \text { and }\left\|P_{m} \phi\right\|_{1} \leq \lambda_{m}\left\|P_{m} \phi\right\| .
$$

Due to the inequality (2.11) the term $b(q, v, q)$ has the following estimate:

$$
2|b(q, v, q)| \leq C\|q\|^{1 / 2}\|\| q\left\|_{1}^{3 / 2}\right\| v\left\|_{1} \leq \frac{C}{\lambda_{m+1}^{1 / 4}}\right\| q\left\|_{1}^{2}\right\| v \|_{1} .
$$

The first term in the right-hand side of (4.8) has the estimate

$$
2|(\theta, q)| \leq \frac{2}{\nu}\|\theta\|_{-1}^{2}+\frac{\nu}{2}\|q\|_{1}^{2}
$$

Employing the inequalities (2.12) and (4.9) we estimate the second term in the righthand side of (4.8) as follows

$$
2|b(v, p, q)| \leq\|v\|_{1}\|p\|_{1}\|q\|^{1 / 2}\|q\|_{1}^{1 / 2} \leq C \lambda_{m} \lambda_{m+1}^{-1 / 4}\|p\|_{1}\left(\|q\|_{1}^{2}+\|v\|_{1}^{2}\right) .
$$

Other terms in the right-hand side of (4.8) can be estimated in a similar way to (4.12). Using estimates (4.10)-(4.12) and the estimates of other terms in the right-hand side of (4.8) we obtain

$$
\frac{d}{d t}\left[\|q\|^{2}+\alpha^{2}\|q\|_{1}^{2}\right]+\frac{\nu}{2}\|q\|_{1}^{2}+\|q\|_{1}^{2}\left(\nu-\frac{C}{\lambda_{m+1}^{1 / 4}}\|v\|_{1}\right) \leq b(t),
$$

where $b(t)$ is satisfying the corresponding condition of Lemma 1.

Let us choose $t_{1}>0$ so large that $\|v(t)\|_{1} \leq M_{1}$, for all $t \geq t_{1}$ and $m$ so that $\mu(m):=$ $\lambda_{m+1}-\left(\frac{C M_{1}}{\nu}\right)^{4}>0$. Then it follows from the last inequality the following relation

$$
\frac{d}{d t}\left[\|q\|^{2}+\alpha^{2}\|q\|_{1}^{2}\right]+\frac{\nu}{2}\|q\|_{1}^{2} \leq b(t), \text { for all } t \geq t_{1},
$$

or

$$
\frac{d}{d t}\left[\|q\|^{2}+\alpha^{2}\|q\|_{1}^{2}\right]+d_{m}\left[\|q\|^{2}+\alpha^{2}\|q\|_{1}^{2}\right] \leq b(t), \text { for all } t \geq t_{1}
$$

where $d_{m}=\frac{\nu}{4} \min \left\{\frac{1}{\alpha^{2}}, \lambda_{m+1}\right\}$.

Thus, due to Lemma 1 the statement of the theorem follows. 
Remark 4.3. Let us observe that the number $m$, for which $\lambda_{m+1}>\frac{C \mathbf{h}^{4}}{\nu^{8} \lambda_{1}^{2}}$ holds, is an upper bound for the minimal number of asymptotically determining modes for weak solutions (i.e., solutions belonging to $\left.L^{\infty}\left(\mathbb{R}^{+} ; H\right) \cap L_{l o c}\left(\mathbb{R}^{+} ; V\right)\right)$ of the initial boundary value problem for the $3 \mathrm{D}$ Navier Stokes equations.

In fact, for weak solutions of NS equations instead of (4.13) we have

$$
\frac{d}{d t}\|q\|^{2}+\lambda_{m+1}^{3 / 4}\left(\nu \lambda_{m+1}^{1 / 4}-C\|v\|_{1}\right)\|q\|^{2} \leq b(t)
$$

and instead of (4.7) we have for weak solutions of NS equations (see, e.g., [11], [10], [20] and [43])

Hence

$$
\limsup _{t \rightarrow \infty} \frac{1}{T} \int_{t}^{t+T}\|v(\tau)\|_{1}^{2} d \tau \leq \frac{\mathbf{h}^{2}}{T \nu^{3} \lambda_{1}^{2}}+\frac{\mathbf{h}^{2}}{\nu^{2} \lambda_{1}} .
$$

$$
\limsup _{t \rightarrow \infty} \frac{1}{T} \int_{t}^{t+T}\|v(\tau)\|_{1} d \tau \leq \frac{\mathbf{h}}{\sqrt{T} \nu^{3 / 2} \lambda_{1}}+\frac{\mathbf{h}}{\nu \sqrt{\lambda_{1}}} .
$$

Thus, the function $a(t):=\lambda_{m+1}^{3 / 4}\left(\nu \lambda_{m+1}^{1 / 4}-C\|v\|_{1}\right)$ satisfies conditions of Lemma 2.1 provided $T$ is large enough and

$$
\lambda_{m+1}>C \frac{\mathbf{h}^{4}}{\nu^{8} \lambda_{1}^{2}}
$$

Different estimates of asymptotic determining modes for weak solutions of 3D NS equations are obtained in [12] (see also [14], [15] and references therein). The estimate obtained in $[12$ involves generalization of the so called mean rate dissipation of energy, per mass and time, i.e. it involves

$$
\varepsilon=\nu \limsup _{t \rightarrow \infty} \frac{1}{t} \int_{0}^{t} \sup _{x \in \Omega}\|\nabla v(x, \tau)\|^{2} d \tau .
$$

For other related results concerning estimates of the number of asymptotic determining degrees of freedom for weak solutions of the 3D NS equations see, e.g., [10], [20] and references therein.

Determining modes on the attractor. Next we give an estimate of determining modes for trajectories on the attractor.

Definition 4.4. A set of modes $\left\{w_{1}, \cdots, w_{m}\right\}$ is called determining on the attractor (in the sense of [31]) if for each two trajectories $v(t)$ and $u(t)$ on the attractor $\mathcal{A}_{1}$ the equality

$$
\left\|P_{m}(v(t)-u(t))\right\|_{1}=0, \text { for all } t \in \mathbb{R}
$$

implies

$$
v(t)=u(t), \quad \forall t \in R .
$$

Let $v$ and $u$ be arbitrary two trajectories in the attractor $\mathcal{A}_{1}$ of (3.1). Then $w=v-u$ satisfies

$$
w_{t}+\alpha^{2} A w_{t}+\nu A w+B(w, v)+B(u, w)=0 .
$$

Taking the inner product of (4.15) with $q=Q_{m} w$ we get

$$
\frac{d}{d t}\left[\|q\|^{2}+\|q\|_{1}^{2}\right]+2 \nu\|q\|_{1}^{2}=-2 b(w, v, q)-2 b(u, w, q) .
$$


Assume that $P_{m} w(t)=0$, for all $t \in \mathbb{R}$, then $Q_{m} w=q$ satisfies

$$
\frac{d}{d t}\left[\|q\|^{2}+\|q\|_{1}^{2}\right]+2 \nu\|q\|_{1}^{2}=2 b(q, v, q) .
$$

Due to (2.11) we have

$$
|2 b(q, v, q)| \leq C\|q\|_{1}^{1 / 2}\|q\|_{1}^{3 / 2}\|v\|_{1}
$$

Noting that on the attractor $\mathcal{A}_{1}$ we have $\|v\|_{1} \leq M_{1}$, we employ the last inequality, and inequality (4.9) to obtain from (4.16)

$$
\frac{d}{d t}\left[\|q\|^{2}+\alpha^{2}\|q\|_{1}^{2}\right]+\nu\|q\|_{1}^{2}+\|q\|^{1 / 2}\|q\|_{1}^{3 / 2}\left(\nu \lambda_{m+1}^{1 / 4}-C M_{1}\right) \leq 0 .
$$

Let us choose $m$, large enough, so that $\lambda_{m+1} \geq\left(\frac{M_{1} C}{\nu}\right)^{4}$. Then (4.18) implies

$$
\frac{d}{d t}\left[\|q\|^{2}+\alpha^{2}\|q\|_{1}^{2}\right]+l_{m}\left[\|q\|^{2}+\alpha^{2}\|q\|_{1}^{2}\right] \leq 0,
$$

where $l_{m}=\frac{\nu}{2} \min \left\{\lambda_{m+1}, \frac{1}{\alpha^{2}}\right\}$.

Finally, we integrate the last inequality and get

$$
\|q(t)\|^{2}+\alpha^{2}\|q(t)\|_{1}^{2} \leq \exp \left[-l_{m}(t-s)\right]\left[\|q(s)\|^{2}+\alpha^{2}\|q(s)\|_{1}^{2}\right] .
$$

Passing to the limit as $s \rightarrow-\infty$ we obtain

$$
\|q(t)\|^{2}+\alpha^{2}\|q(t)\|_{1}^{2}=0, \text { for all } t \in \mathbb{R} .
$$

Thus, the following theorem is true.

Theorem 4.5. Let $v$ and $u$ be two solutions of the problem (1.1)-(1.3) from the attractor $\mathcal{A}_{1}$. Assume that $P_{m}(u(t))=P_{m}(v(t)), \forall t \in \mathbb{R}$, where $m$ is so that

$$
\lambda_{m+1} \geq C \frac{\|h\|_{-1}^{4}}{\alpha^{4} \nu^{8} d_{1}^{2}} .
$$

Then $v(t)=u(t)$, for allt $\in \mathbb{R}$.

\section{Estimates of Dimensions of the Global Attractor}

In this section we show the differentiability of the semigroup with respect to the initial data. This is to prepare for implementing Theorem 2.4 in order to estimate the dimension of the global attractor.

Theorem 5.1. Let $u_{0}$ and $v_{0}$ be two elements of $V$. Then there is a constant $K=$ $K\left(\left\|u_{0}\right\|_{1},\left\|v_{0}\right\|_{1}\right)$ such that

$$
\left\|S(t) v_{0}-S(t) u_{0}-\Lambda(t)\left(v_{0}-u_{0}\right)\right\|_{1} \leq K\left\|v_{0}-u_{0}\right\|_{1}^{2}
$$

where the linear operator $\Lambda(t): V \rightarrow V$, for $t>0$ is the solution operator of the problem

$$
\xi_{t}+\alpha^{2} A \xi_{t}+A \xi+B(\xi, v)+B(v, \xi)-B(\xi, \xi)=0, \xi(0)=v_{0}-u_{0},
$$

and $v(t)=S(t) v_{0}$. That is, for every $t>0$, the map $S(t) v_{0}$, as a map $S(t): V \rightarrow$ $V$ is Fréchet differentiable with respect to the initial data, and its Fréchet derivative $D_{v_{0}}\left(S(t) v_{0}\right) w_{0}=\Lambda(t) w_{0}$. 
Proof. It is easy to see that the function $\eta(t):=v(t)-u(t)-\xi(t)=S(t)\left(v_{0}-u_{0}\right)-\xi(t)$ satisfies

$$
\eta_{t}+\alpha^{2} A \eta_{t}+\nu A \eta+B(\eta, v)+B(v, \eta)-B(w, w)=0
$$

where $w=v-u$. Taking the inner product of the last equation with $\eta$ we obtain

$$
\frac{d}{d t}\left[\|\eta\|^{2}+\alpha^{2}\|\eta\|_{1}^{2}\right]+2 \nu\|\eta\|_{1}^{2}=-2 b(\eta, v, \eta)-2 b(w, w, \eta) .
$$

By using inequalities (3.5) and (2.5) and Young's inequality we can estimate the terms in the right-hand side of (5.3) as follows.

By (2.11) we have

$$
|2 b(\eta, v, \eta)| \leq C\|v\|_{1}\|\eta\|^{1 / 2}\|\eta\|_{1}^{3 / 2} \leq C M_{1}\|\| \eta\left\|^{1 / 2}\right\| \eta \|_{1}^{3 / 2} \leq \frac{C M_{1}}{4}\left(\|\eta\|^{2}+3\|\eta\|_{1}^{2}\right) .
$$

By (2.10)

$$
|2 b(w, w, \eta)|=|2 b(w, \eta, w)| \leq C \lambda_{1}^{-\frac{1}{2}}\|\eta\|_{1}\|w\|_{1}^{2} \leq \nu\|\eta\|_{1}^{2}+\frac{C}{4 \nu \lambda_{1}}\|w\|_{1}^{4} .
$$

Hence, we obtain from (5.3)

$$
\frac{d}{d t}\left[\|\eta\|^{2}+\alpha^{2}\|\eta\|_{1}^{2}\right] \leq \frac{C M_{1}}{4}\left(\|\eta\|^{2}+3\|\eta\|_{1}^{2}\right)+\frac{C}{4 \nu \lambda_{1}}\|w\|_{1}^{4}
$$

The function $w(t)=v(t)-u(t)=S(t) v_{0}-S(t) u_{0}$ satisfies

$$
w_{t}+\alpha^{2} A w_{t}+\nu A w+B(w, v)+B(v, w)-B(w, w)=0, w(0)=v_{0}-u_{0}:=w_{0} .
$$

Taking the inner product of the last equation with $w$, and using (2.13) and $\left(E_{2}\right)$ we obtain

$$
\begin{aligned}
\frac{d}{d t}\left[\|w\|^{2}+\alpha^{2}\|w\|_{1}^{2}\right]+2 \nu\|w\|_{1}^{2}=2 b(w, v, w) \leq 2 C \lambda_{1}^{1 / 4}\|v\|_{1}\|w\|_{1}^{2} \leq \\
\kappa_{1}\|v\|_{1}\left[\|w\|^{2}+\alpha^{2}\|w\|_{1}^{2}\right]
\end{aligned}
$$

where $k_{1}=2 C \lambda_{1}^{1 / 4} \alpha^{-4}\left[\|v(0)\|^{2}+\alpha^{2}\|v(0)\|_{1}^{2}+\left(1 / \nu d_{0}\right)\|h\|_{-1}^{2}\right]^{1 / 2}$. Integrating last inequality we get

$$
\|w(t)\|_{1}^{2} \leq\left(1+\frac{1}{\lambda_{1} \alpha^{2}}\right)\|w(0)\|_{1}^{2} \exp \left(\kappa_{1} t\right) .
$$

It follows from (5.4) and (5.5) that

$$
\frac{d}{d t}\left[\|\eta\|^{2}+\alpha^{2}\|\eta\|_{1}^{2}\right] \leq A_{1}\left[\|\eta\|^{2}+\alpha^{2}\|\eta\|_{1}^{2}\right]+A_{2}\|w(0)\|_{1}^{4} \exp \left(2 \kappa_{1} t\right) .
$$

Integrating and using Gronwall's inequality :

$$
\|\eta(t)\|_{1}^{2} \leq A(t)\|w(0)\|_{1}^{4},
$$

where $A(t):=\frac{A_{2}}{2 \kappa_{1} \alpha^{2}} \exp \left(2 \kappa_{1}+A_{1}\right) t$. So we have

$$
\frac{\|v(t)-u(t)-\xi(t)\|_{1}}{\left\|v_{0}-u_{0}\right\|_{1}} \leq \sqrt{A(t)}\left\|v_{0}-u_{0}\right\|_{1} .
$$


Thus the differentiability of $S(t)$ with respect to the initial data follows.

We rewrite (3.1) in the following form

$$
\hat{v}_{t}=-\frac{\nu}{\alpha^{2}} \hat{v}+\frac{\nu}{\alpha^{2}} G^{-2} \hat{v}-G^{-1} B\left(G^{-1} \hat{v}, G^{-1} \hat{v}\right)+G^{-1} h,
$$

where $G^{2}=I+\alpha^{2} A$, and $\hat{v}=G v$.

The equation of linear variations corresponding to (5.6) has the form

$$
w_{t}=L(t) w,
$$

where

$$
L(t) w:=-\frac{\nu}{\alpha^{2}} w+\frac{\nu}{\alpha^{2}} G^{-2} w-G^{-1} B\left(G^{-1} w, G^{-1} \hat{v}\right)-G^{-1} B\left(G^{-1} \hat{v}, G^{-1} w\right) .
$$

Now we consider the quadratic form

$$
(L(t) w, w)=-\frac{\nu}{\alpha^{2}}\|w\|^{2}+\frac{\nu}{\alpha^{2}}\left\|G^{-1} w\right\|^{2}-b\left(G^{-1} w, G^{-1} \hat{v}, G^{-1} w\right) .
$$

By using inequality (2.11) and the inequality $\left\|G^{-1} u\right\|_{1} \leq \frac{1}{\alpha}\|u\|$ we get

$$
\left|b\left(G^{-1} w, G^{-1} \hat{v}, G^{-1} w\right)\right| \leq \frac{1}{\alpha^{5 / 2}}\left\|G^{-1} w\right\|^{1 / 2}\|w\|^{3 / 2}\|\hat{v}\| .
$$

Employing Young's inequality with $p=4 / 3, \epsilon=2 \nu /\left(3 \alpha^{2}\right)$, and the fact that on the global attractor $\mathcal{A}_{1}$ the estimate $\|\hat{v}\| \leq\left(\lambda_{1}+\alpha^{2}\right)^{1 / 2} M_{1}$ holds, we obtain

$$
\left|b\left(G^{-1} w, G^{-1} \hat{v}, G^{-1} w\right)\right| \leq \frac{\nu}{2 \alpha^{2}}\|w\|^{2}+\frac{C\left(\lambda_{1}+\alpha^{2}\right)^{2} M_{1}^{4}}{\nu^{3} \alpha^{4}}\left\|G^{-1} w\right\|^{2} .
$$

Due to the last inequality the quadratic form $(L(t) w, w)$ has the following estimate

$$
(L(t) w, w) \leq-\frac{\nu}{2 \alpha^{2}}\|w\|^{2}+\left(\frac{\nu}{\alpha^{2}}+\frac{C\left(\lambda_{1}+\alpha^{2}\right)^{2} M_{1}^{4}}{\nu^{3} \alpha^{4}}\right)\left\|G^{-1} w\right\|^{2}
$$

Thus, we can use Theorem 2.4 to get the desired estimate for the fractal dimension of the attractor $\mathcal{A}_{1}$

$$
d_{f}\left(\mathcal{A}_{1}\right) \leq C \frac{\left(\lambda_{1}+\alpha^{2}\right)^{2} M_{1}^{4}}{\nu^{4} \alpha^{2}}+2 \leq C \frac{\left(\lambda_{1}+\alpha^{2}\right)^{2}\|h\|_{-1}^{4}}{\nu^{8} \alpha^{6} d_{1}^{2}}+2 .
$$

We recall that $M_{1}=\frac{2}{\nu \alpha \sqrt{d_{1}}}\|h\|_{-1}, \quad d_{1}=\frac{1}{2} \min \left\{\alpha^{-2}, \lambda_{1}\right\}$. Let us note that in our situation

$$
\bar{h}_{0}(t)=\frac{\nu}{2 \alpha^{2}}, s_{0}=0, s_{1}=-1, \bar{h}_{s_{1}}(t)=\frac{\nu}{\alpha^{2}}+\frac{C\left(\lambda_{1}+\alpha^{2}\right)^{2} M_{1}^{4}}{\nu^{3} \alpha^{4}}
$$

and $\bar{h}_{s_{k}}(t)=0, k \geq 2$. 


\section{The INVISCID Limit}

Here we show that when $\nu \rightarrow 0$ the weak solution of the initial boundary value problem for the NSV system, i.e. of the problem (1.1)-(1.3), is tending to the weak solution of the initial boundary value problem for the inviscid simplified Bardina model

$$
\begin{array}{r}
u_{t}-\alpha^{2} \Delta u_{t}+(u \cdot \nabla) u+\nabla p=f, \quad x \in \Omega, t>0, \\
\nabla \cdot u=0, \quad x \in \Omega, t>0, \\
u(x, 0)=v_{0}(x), \quad x \in \Omega ; \quad u(x, t)=0, x \in \partial \Omega, t>0 .
\end{array}
$$

The problem of existence and uniqueness of solutions of the initial boundary value problem, with periodic boundary conditions, for the 3D viscous and inviscid simplified Bardina models is studied in [5]. In particular, it is shown in [5] that the problem (6.1)(6.2) has a unique solution $u \in C^{1}(\mathbb{R} ; V)$, for initial value $u_{0} \in V$.

Applying to (6.1) the Helmholtz-Leray operator $P$ we obtain the equivalent functional differential equation

$$
\begin{gathered}
u_{t}+\alpha^{2} A u_{t}+B(u, u)=h, \\
u(0)=v_{0} .
\end{gathered}
$$

Let $v(t)$ be the solution of (6.1) with initial $v(0)=v_{0} \in V$. Denote by $w=v-u$. Then $w$ satisfies the relation

$$
\begin{gathered}
w_{t}+\alpha^{2} A w_{t}+B(w, v)+B(u, w)=-\nu A v, \\
w(0)=0,
\end{gathered}
$$

which holds in the space $V^{\prime}$. Taking the action of (6.5) on $w$, which belongs to $V$, and using a Lemma of Lions-Magenes concerning the derivative of functions with values in Banach space (cf. Lemma 1.2 Chap. III-p.169-[44), we obtain

$$
\frac{d}{d t}\left[\|w\|^{2}+\alpha^{2}\|w\|_{1}^{2}\right]=-2 \nu(\nabla v, \nabla w)-2 b(w, v, w) .
$$

For the first term in the right-hand side we have

$$
|2 \nu(\nabla v, \nabla w)| \leq \nu^{2}\|v\|_{1}^{2}+\|w\|_{1}^{2} .
$$

The second term we estimate by using the inequality (2.13)

$$
|2 b(w, v, w)| \leq C \lambda_{1}^{1 / 4}\|v\|_{1}\|w\|_{1}^{2} .
$$

Utilizing last two inequalities in (6.7) we get

$$
\begin{gathered}
\frac{d}{d t}\left[\|w\|^{2}+\alpha^{2}\|w\|_{1}^{2}\right] \leq \nu^{2}\|v\|_{1}^{2}+\left(1+C \lambda_{1}^{1 / 4}\|v\|_{1}\right)\|w\|_{1}^{2} \leq \\
\nu^{2}\|v\|_{1}^{2}+\alpha^{-2}\left(1+2 C \lambda_{1}^{1 / 4}\|v\|_{1}\right)\left[\|w\|^{2}+\alpha^{2}\|w\|_{1}^{2}\right] .
\end{gathered}
$$

Integrating the last inequality and using the standard Gronwall's lemma we get the estimate

$$
\|w(t)\|^{2}+\alpha^{2}\|w(t)\|_{1}^{2} \leq \nu^{2} \int_{0}^{t}\|v(\tau)\|_{1}^{2} d \tau \exp \left(\frac{t}{\alpha^{2}}+\frac{2 C \lambda_{1}^{1 / 4}}{\alpha^{2}} \int_{0}^{t}\|v(\tau)\|_{1} d \tau\right) .
$$


Next we show that on each finite interval $[0, T]$ we can estimate $\|v\|_{1}$ by a constant depending only on $\left\|v_{0}\right\|,\left\|v_{0}\right\|_{1}$ and the parameter $\alpha$. Indeed, (3.3) implies that

$$
\frac{d}{d t}\left[\|v(t)\|^{2}+\alpha^{2}\|v(t)\|_{1}^{2}\right] \leq \alpha^{-2}\|h\|_{-1}^{2}+\alpha^{2}\|v(t)\|_{1}^{2} .
$$

Integrating the last inequality over $(0, t)$ with respect to time variable we obtain

$$
\|v(t)\|^{2}+\alpha^{2}\|v(t)\|_{1}^{2} \leq\left\|v_{0}\right\|^{2}+\alpha^{2}\left\|v_{0}\right\|_{1}^{2}+t \alpha^{-2}\|h\|_{-1}^{2}+\int_{0}^{t}\left[\|v(\tau)\|^{2}+\alpha^{2}\|v(\tau)\|_{1}^{2}\right] d \tau
$$

By using the Gronwall inequality we get

$$
\|v(t)\|_{1}^{2} \leq D_{T} e^{T}, \text { for all } t \in[0, T] .
$$

Here $D_{T}:=\alpha^{-2}\left[\left\|v_{0}\right\|^{2}+\alpha^{2}\left\|v_{0}\right\|_{1}^{2}+T \alpha^{-2}\|h\|_{-1}^{2}\right]$.

Hence (6.8) implies

$$
\|w(t)\|^{2}+\alpha^{2}\|w(t)\|_{1}^{2} \leq \nu^{2} T D_{T} e^{T} \exp \left(\alpha^{-2} T+2 C \alpha^{-2} \lambda^{1 / 4} T D_{T}^{1 / 2} e^{T / 2}\right) .
$$

Remark 6.1. The problem of convergence of solutions of the NSV equations to solutions of NS equations as $\alpha \rightarrow 0$ was studied in [39]. It is shown in [39] that strong solutions of the NSV equations converge to strong solutions of the NS equations as $\alpha \rightarrow 0$, under specified smallness conditions on the initial data of the problem.

Remark 6.2. The results obtained in this paper are valid also for the solutions of the initial boundary value problem for the 3D NSV equations with periodic boundary conditions.

Finally we would like to notice that the results reported here can be extended to other similar equations, a subject of future work. For instance, for the 3D equations of motion of Kelvin-Voight fluids of order $L \geq 1$ :

$$
\begin{gathered}
v_{t}+(v \cdot \nabla) v-\mu_{0} \Delta v_{t}-\mu_{1} \Delta v-\sum_{l=1}^{L} \beta_{l} \Delta u_{l}+\nabla p=f, \\
\partial_{t} u_{l}+\alpha_{l} u_{l}-v=0, \quad l=1, \ldots, L .
\end{gathered}
$$

where $\mu_{0}, \mu_{1}, \beta_{l}, \alpha_{l}>0, l=1, \ldots, L$. Also for the generalized Benajamin-Bona-Mahony (GBBM) equation:

$$
u_{t}-\alpha^{2} \Delta u_{t}+\nu \Delta u+\nabla \cdot \vec{F}(u)=h,
$$

where a smooth vector field $\vec{F}(u)$ satisfies the growth condition

$$
|\vec{F}(u)| \leq C\left(1+|u|^{2}\right)
$$

The problem of existence of a finite dimensional global attractor and estimates for the number of determining modes on the global attractor of Kelvin-Voight fluids of order $L \geq 1$ is established in [27]. In [45] the existence of a finite dimensional global attractor is established for 1D GBBM equation under periodic boundary conditions. Existence of a finite dimensional global attractor for 3D GBBM under periodic boundary conditions is 
proved in [6]. In [42] it was shown the existence of the global attractor for GBBM equation in $H^{1}\left(\mathbb{R}^{3}\right)$. Moreover, the existence of a global attractor for a similar two-dimensional model describing the motion of a second-grade fluid is established in [35].

Acknowledgements. The work of the V. K. Kalantarov was supported in parts by The Scientific and Research Council of Turkey, grant no. 106T337. The work of E. S. Titi was supported in parts by the NSF grant no. DMS-0504619, the ISF grant no. 120/6, and the BSF grant no. 2004271.

\section{REFERENCES}

[1] R. A. Adams, Sobolev Spaces, Academic Press, New York, 1975.

[2] A. V. Babin, M. I. Vishik, Attractors of Evolution Equations, North-Holland, Amsterdam, 1992..

[3] J. Bardina, J. Ferziger, and W. Reynolds, Improved subgrid scale models for large eddy simulation, American Institute of Aeronatics and Astronautics, 80 (1980), 80-1357.

[4] L. C. Berselli, T. Iliescu, W. J. Layton, Mathematics of Large Eddy Simulation of Turbulent Flows, Springer, Scientific Computation, New York, (2006).

[5] Y. Cao, E.M. Lunasin, and E.S. Titi, Global well-posedness of the three dimensional viscous and inviscid simplified Bardina turbulence models, Communications in Mathematical Sciences, 4 (2006), no.4, 823-84.

[6] A. O. Çelebi, V. K. Kalantarov, M. Polat, Attractors for the generalized Benjamin-Bona-Mahony equation. J. Differential Equations 157 (1999), no. 2, 439-451.

[7] I. D. Chueshov, The theory of functionals that uniquely determine the asymptotic dynamics of infinite-dimensional dissipative systems, Russian Math. Surveys 53 (1998), no. 4, 731-776.

[8] B. Cockburn, D. Jones and E. S. Titi, Determining degrees of freedom for nonlinear dissipative equations, C. R. Acad. Sci. Paris Sér. I Math. 321 (1995), no. 5, 563-568.

[9] B. Cockburn, D. Jones and E. S. Titi, Estimating the number of asymptotic degrees of freedom for nonlinear dissipative systems, Math. Comput. 66 (1997), 1073-87.

[10] P. Constantin, C. R. Doering and E. S. Titi, Rigorous estimates of small scales in turbulent flows, Jour. Math. Physics 37 (1996), 6152-6156.

[11] P. Constantin and C. Foias, Navier-Stokes Equations. Chicago Lectures in Mathematics. University of Chicago Press, Chicago, IL, 1988.

[12] P. Constantin, C. Foias, O. P. Manley, and R. Temam, Determining modes and fractal dimension of turbulent flows, J. Fluid Mech. 150 (1985), 427-440.

[13] P. Constantin, C. Foias, B. Nicolaenko and R. Temam, Integral Manifolds and Inertial Manifolds for Dissipative Partial Differential Equations. Applied Mathematical Sciences, 70. Springer-Verlag, New York, 1989.

[14] P. Constantin, C. Foias, R. Temam, Attractors Representing Turbulent Flows, Mem. Amer. Math. Soc. 53, no. 314, 1985.

[15] C. Foias,O. Manley, R. Rosa, R. Temam, Navier-Stokes Equations and Turbulence, Cambridge University Press, New York, 2001.

[16] C. Foias, O. P. Manley, R. Temam and Y. M. Tréve, Asymptotic analysis of the Navier-Stokes equations. Phys. D 9 (1983), no. 1-2, 157-188.

[17] C. Foias, G. Prodi, Sur le comportement global des solutions non-stationnaires des quations de Navier-Stokes en dimension 2, Rend. Sem. Mat. Univ. Padova 39 (1967), 1-34.

[18] C. Foias and E. S. Titi, Determining nodes, finite difference schemes and inertial manifolds, Nonlinearity 4 (1991), 135-153.

[19] J. K. Hale, Asymptotic Behavior of Dissipative Systems. Mathematical Surveys and Monographs, 25. American Mathematical Society, Providence, RI, 1988. 
[20] M. J. Holst, E. S. Titi, Determining projections and functionals for weak solutions of the NavierStokes equations, Recent Developments in Optimization Theory and Nonlinear Analysis (Jerusalem, 1995), 125-138, Contemp. Math., 204, Amer. Math. Soc., Providence, RI, 1997.

[21] A. A. Ilyin, Attractors for Navier-Stokes equations in domains with finite measure, Nonlinear Anal. 27 (1996), 605-616.

[22] A. A. Ilyin and E. S. Titi, Sharp estimates for the number of degrees of freedom for the damped driven 2D Navier-Stokes equations, Journal of Nonlinear Sciences, J. Nonlinear Sci. 16 (2006), no.3, 233-253.

[23] D. A. Jones and E. S. Titi, Determining finite volume elements for the 2D Navier-Stokes equations, Physica D 60 (1992), 165-174.

[24] D. A. Jones and E. S. Titi, Upper bounds on the number of determining modes, nodes, and volume elements for the Navier-Stokes equations, Indiana Univ. Math. J. 42 (1993), 875-887.

[25] V. K. Kalantarov, Attractors for some nonlinear problems of mathematical physics, Zap. Nauchn. Sem. Leningrad. Otdel. Mat. Inst. Steklov. (LOMI) 152 (1986), 50-54.

[26] V.K. Kalantarov, Global Behavior of Solutions of Nonlinear Equations of Mathematical Physics of Classical and Non-classical Type, Postdoctoral Thesis, St. Petersburg, 1988.

[27] N. A. Karazeeva, A.A. Kotsiolis, A.P. Oskolkov, Dynamical systems generated by initial-boundary value problems for equations of motion of linear viscoelastic fluids, Proc. Steklov Inst. Math. 1991, no. 3, 73-108.

[28] B. Khouider, E. S. Titi, An Inviscid regularization for the surface quasi-geostrophic equation, Comm. Pure Appl. Math. (to appear). arXiv math/0702067v1 [math.AP].

[29] W. D. Henshaw, H. O. Kreiss, J. Yström, Numerical experiments on the interaction between the large and small-scale motions of the Navier-Stokes equations, Multi- scale Model. Simul. 1 (2003), 119-149.

[30] O. A. Ladyzhenskaya, On the dynamical system generated by the NavierStokes equations, Zap. Nauchn. Sem. LOMI 27 (1972), 91-114

[31] O. A. Ladyzhenskaya, The Mathematical Theory of Viscous Incompressible Flow, New York: Gordon and Breach Science publishers (1963).

[32] O. A. Ladyzhenskaya, Attractors for Semigroups and Evolution Equations, Lezioni Lincee, Cambridge University Press, Cambridge, 1991.

[33] O. A. Ladyzhenskaya, V. A. Solonnikov, N. N. Uraltseva, Linear and Quasilinear Equations of Parabolic Type, Nauka, 1967.

[34] R. Layton and R. Lewandowski, On a well-posed turbulence model, Discrete and Continuous Dyn. Sys. B, 6 (2006), 111-128.

[35] I. Moise, R. Rosa, X. Wang, Attractors for non-compact semigroups via energy equations, Nonlinearity 11 (1998), no. 5, 1369-1393.

[36] E. Olson, E. S. Titi, Determining modes for continuous data assimilation in $2 D$ turbulence, J. Statist. Phys. 113 (2003), no. 5-6, 799-840.

[37] E. Olson and E. S. Titi, Determining modes and Grashof number in 2D turbulence - A numerical case study, Preprint (2007).

[38] A. P. Oskolkov, The uniqueness and solvability in the large of boundary value problems for the equations of motion of aqueous solutions of polymers, Zap. Naučn. Sem. Leningrad. Otdel. Mat. Inst. Steklov. (LOMI) 38 (1973), 98-136.

[39] A. P. Oskolkov, A certain nonstationary quasilinear system with a small parameter, that regularizes the system of Navier-Stokes equations, in "Problems of Mathematical Analysis, No. 4: Integral and Differential Operators. Differential Equations", 143 (1973) pp. 78-87, St. Petersburg Univ., St. Petersburg.

[40] A. P. Oskolkov, On the theory of Voight fluids, Zap. Nauchn. Sem. LOMI, 96 (1980), 233-236.

[41] J. Robinson, Infinite-dimensional Dynamical Systems, Cambridge University Press "Texts in Applied Mathematics" Series (2001).

[42] M. Stanislavova, A. Stefanov, B. Wang, Asymptotic smoothing and attractors for the generalized Benjamin-Bona-Mahony equation on $R^{3}$, J. Differential Equations 219 (2005), no. 2, 451-483. 
[43] R. Temam, Infinite-dimensional Dynamical Systems in Mechanics and Physics, Springer, New York, 1997.

[44] R. Temam, Navier-Stokes Equations, Theory and Numerical Analysis, 3rd revised edition, NorthHolland, 2001.

[45] B. Wang, W. Yang, Finite-dimensional behaviour for the Benjamin-Bona-Mahony equation, J. Phys. A 30 (1997), no. 13, 4877-4885.

(V.K.Kalantarov) Department of mathematics, Koç University, RUMELIFENERI Yolu, SARIYER 34450

SARIYER, Istanbul, TURKey

E-mail address: vkalantarov@ku.edu.tr

(E.S.Titi) Department of mathematics and Department

of Mechanical and Aerospace Engineering,

UNIVERSITY OF CALIFORNIA

IRvine, CALIFORNiA 92697, USA.

Also:

Department of Computer Science and Applied Mathematics

Weizmann Institute of Science

REHOVOT, 76100, ISRAEL

E-mail address: etiti@math.uci.edu

E-mail address: edriss.titi@weizmann.ac.il 